\title{
Reinventando los espacios como lugares de vida y paz una estrategia desde la lúdica para sectores vulnerables ${ }^{1}$
}

\author{
Reinventing spaces as places of life and peace, a playful strategy for vulnerable sectors
}

Greys, Nuñez-Ríos ${ }^{2}$

https://orcid.org/0000-0001-6426-2729

Kadry, García- Mendoza ${ }^{3}$

https://orcid.org/0000-0002-4090-8094

Judith, Castillo-Martelo ${ }^{4}$

(iD) https://orcid.org/0000-0002-4956-1453

Nevis, Niño-Jiménez ${ }^{5}$

(D) https://orcid.org/0000-0002-8254-9368

Universidad de La Costa, Colombia

\section{RESUMEN}

El presente artículo es producto de una investigación acción - participativa, cuyo objetivo consistió en intervenir a una comunidad de un sector vulnerable y marginal de la ciudad de Barranquilla, en los aspectos económicos, social y físico con estrategias lúdico - pedagógicas para transformar los espacios de estas zonas expuestas en lugares de vida y de paz, que desde las dinámicas sociales inviten al fortalecimiento de la participación y las competencias ciudadanas, la inteligencia emocional y la cultura ambiental. Se abordó desde un paradigma cualitativo con un diseño no experimental y de campo, de alcance descriptivo transversal. Tras la intervención se encontró que la comunidad se motiva para participar en las actividades que propendan por generar una cultura de paz para el encuentro y disfrute colectivo donde sus actores reinventan sus relaciones y transforman las categorías fundamentales que rigen su vida cotidiana. Se concluye que estas intervenciones son una oportunidad para acercar a la población al desarrollo de sus competencias ambientales, sensibilizarlos sobre el impacto ambiental y su compromiso con el desarrollo sostenible; así como potenciar su desarrollo afectivo, generar transformación de historias de vida articulando la lectura y la narración oral para impactar en la relación hombre - sociedad- naturaleza.

Palabras claves: Creación cultural, cultura de paz, desarrollo afectivo, educación ciudadana, grupo vulnerable.

\section{ABSTRACT}

This article is the product of an action-participatory research, the objective of which was to intervene in a community in a vulnerable and marginal sector of the city of Barranquilla, in economic, social and physical aspects, with playful-pedagogical strategies to transform the spaces of these exposed areas in places of life and peace, which from the social dynamics invite the strengthening of citizen participation and competences, emotional intelligence and environmental culture. It was approached from a qualitative paradigm with a non-experimental and field design, with a transversal descriptive scope. After the intervention, it was found that the community is motivated to participate in activities that seek to generate a culture of peace for the encounter and collective enjoyment where its actors reinvent their relationships and transform the fundamental categories that govern their daily lives. It is concluded that these interventions are an opportunity to bring the population closer to the development of their environmental competencies, sensitize them to the environmental impact and their commitment to sustainable development; as well as enhancing their affective development, generating transformation of life stories articulating reading and oral narration to impact the man-society-nature relationship

Keywords: Cultural creation, civic education, culture of peace, emotional development, vulnerable groups.

Recibido: 12 de Octubre 2019 - Aceptado: 12 de Febrero 2020 - Corregido: 15 de Marzo 2020

Cómo referenciar este artículo:

Nuñez-Ríos, G., García- Mendoza, K., Castillo-Martelo, J. \& Niño-Jiménez, N. (2020).Reinventando los espacios como lugares de vida y paz una estrategia desde la lúdica para sectores vulnerables. Politica Globalidad y Ciudadanía, 128-148. Recuperado de http://revpoliticas.uanl.mx/index.php/RPGyC/article/view/151

\footnotetext{
1 Proyecto de investigación "Reinventando espacios como lugares de vida y paz: Estrategia para fortalecer la relación hombre-sociedad-naturaleza a través de la Cultura Ambiental y la Promoción de Lectrua", Financiado por Universidad de La Costa.

2 Magister en Educación por la Universidad Autónoma del Caribe, Profesor medio tiempo, Universidad de la Costa (CUC) Email: gnunez4@ cuc.edu.co

3 Magister en Educación por la Universidad de la Costa, Profesor tiempo completo, Universidad de la Costa (CUC), Email: kgarcia@cuc. edu.co.

4 Magister en Educación por la Universidad San Buenaventura, Directora del programa de Licenciatura en Educación Básica Primaria de la Universidad de la Costa (CUC), Email: jcantill39@cuc.edu.co

5 Especialista en ética y Pedagogía de la Universidad Juan de Castellanos, Profesor Catedrático, Universidad de la Costa (CUC), Email: nnino2@cuc.edu.co
} 


\section{1.- INTRODUCCION}

"Leer es una opción inteligente, difícil, exigente, pero gratificante. Nadie lee o estudia auténticamente si no asume, frente al texto o al objeto de la curiosidad, la forma crítica de ser o de estar siendo sujeto de esa curiosidad, sujeto de lectura, sujeto del proceso de conocer en el que se encuentra. Leer es procurar o buscar crear la comprensión de lo leído; de ahí la importancia de la enseñanza correcta de la lectura y de la escritura, entre otros puntos fundamentales".

Paulo Freire. Cartas a quien pretende enseñar, 1994

El presente artículo de investigación, es producto de la intervención realizada en el sector de Islita - caño de la ciudad de Barranquilla, por parte de las estudiantes de V semestre del programa de Licenciatura en Educación Básica primaria de la Universidad de la Costa (CUC), a partir de la reflexión colectiva que surge de la pregunta: ¿Cómo intervenir espacios vulnerables para transformarlos en lugares de vida y de paz desde la cultura ambiental y la participación ciudadana?

El objetivo principal de esta experiencia educativa es intervenir el sector vulnerable de islita - caño para reinventar los espacios como lugares de vida y de paz que fortalezcan la relación hombre- sociedad- naturaleza a través de la cultura ambiental y el desarrollo de competencias emocionales.

Para alcanzar este propósito se han establecido los siguientes objetivos específicos:

Implementar la lúdica como dinamizador social para la apropiación de conocimientos y competencias relacionadas con cultura y la paz abordando problemas de tipo social, cultural, económico y de memoria histórica que ayudan a reconstruir el tejido social.

Fortalecer las competencias emocionales, autoconocimiento, autorregulación y empatía, a través de la identificación de las emociones, reconociendo su importancia en la vida diaria.

Con estos objetivos, se plantean estrategias lúdicas para que a través del juego se puedan proyectar deseos, miedos, emociones que no se pueden expresar fácilmente con palabras y permite la evolución de las dimensiones del ser, aprendiendo a vivir y convivir con el otro, aceptando las diferencias, particularidades e individualidades (Núñez Ríos, G., Márquez Núñez, E., Guerrero Cuentas, H., Magdaniel Durán, M., \& Silvera Goenaga, B. (2019) que permitan reinventar los espacios como lugares de vida y de paz, robustezcan las relaciones recíprocas de dar y recibir entre los actores, asumiendo que la paz no es un estado perceptible únicamente de dejación de armas, sino la armonía perfecta que permite reconstruir la sociedad, promover la

Revista Política, Globalidad y Ciudadanía, Vol. 6. No. 12, Julio - Diciembre 2020 Universidad Autónoma de Nuevo León, Monterrey, México, ISSN 2395-8448. 128-148. http://revpoliticas.uanl.mx/index.php/RPGyC/article/view/151 
prosperidad general y garantizar de la efectividad de los principios, derechos y deberes consagrados en la Constitución. (Decreto 1038 del 2015).

Por lo anterior cobra vital importancia el desarrollo de la dimensión afectiva, específicamente de las competencias emocionales, pues se busca el desarrollo de habilidades y actitudes que les permita a los participantes gestionar sus propias emociones y conocer las emociones de los demás, teniendo en cuenta que educar las emociones facilita la adquisición de un espíritu crítico, la resolución de conflictos de manera pacífica y la adquisición de habilidades sociales, de acuerdo con lo anterior estas habilidades conducen a una vida plena, permiten regular, interactuar con los otros, no intelectuales, pero son una meta educativa. Pantoja y Aguaded (2015).

Cabe resaltar la importancia que tiene el desarrollo de las competencias emocionales en las primeras etapas, pues juegan un papel esencial para la vida, además, constituyen la base o condición necesaria para el progreso del niño en las diferentes dimensiones de su desarrollo.

Se pretende, por tanto, promover un cambio de mentalidad para pasar de la idea de despojar la naturaleza" a la idea de entenderse con la naturaleza. Si el hombre no fuera un ser racional, el hecho de relacionarse de manera abusiva con la naturaleza pudiera pasar de forma desapercibida, sin medir los daños y consecuencias que hoy se viven en el mundo. Pero como el hombre es un ser pensante, cultural, político y social, sigue siendo posible educarlo en los valores, las creencias y las actitudes que sean los elementos fundamentales que le den sentido al comportamiento ambiental.

Además de lo anterior, se articula con la participación ciudadana, lo cual le permite proponer políticas públicas y ambientales que ayuden a controlar los abusos de la humanidad hacia la naturaleza y se puedan generar proyectos de preservación medio ambiental.

Se pretende por tanto, la transformación en los sectores y poblaciones vulnerables, que ayuden generar una conciencia sobre la conservación del medio ambiente desde los principios sostenibles, la participación ciudadana y las relaciones entre los hombres de manera armónica donde la concientización cultural establezca el impacto sobre las condiciones naturales y los procesos ecológicos con el fin de generar espacios de reflexión útiles que puedan extrapolarse en todos los contextos.

En consecuencia, se intenta desde estos espacios formar un ciudadano de mundo que construye una ciudad diferente, que lleva en sus arraigos una cultura ambiental necesaria para cambiar y transformar la relación del hombre con la naturaleza. Un hombre que vivencia en la sociedad los valores de justicia, dignidad, solidaridad, entre otros que lo ayudan a reestablecer las relaciones armónicas con los demás y donde la participación converge para generar políticas de desarrollo sostenible.

En este orden de ideas, se propende por la construcción de una cultura ambiental a partir 
de la realidad circundante en las zonas olvidadas y marginadas fortaleciendo la concientización de esta desde los procesos lúdicos y reflexivos para que se refleje en los espacios sociales y culturales, como pilar del desarrollo sostenible.

Ilustración 1. Ubicación espacial población de la isla.

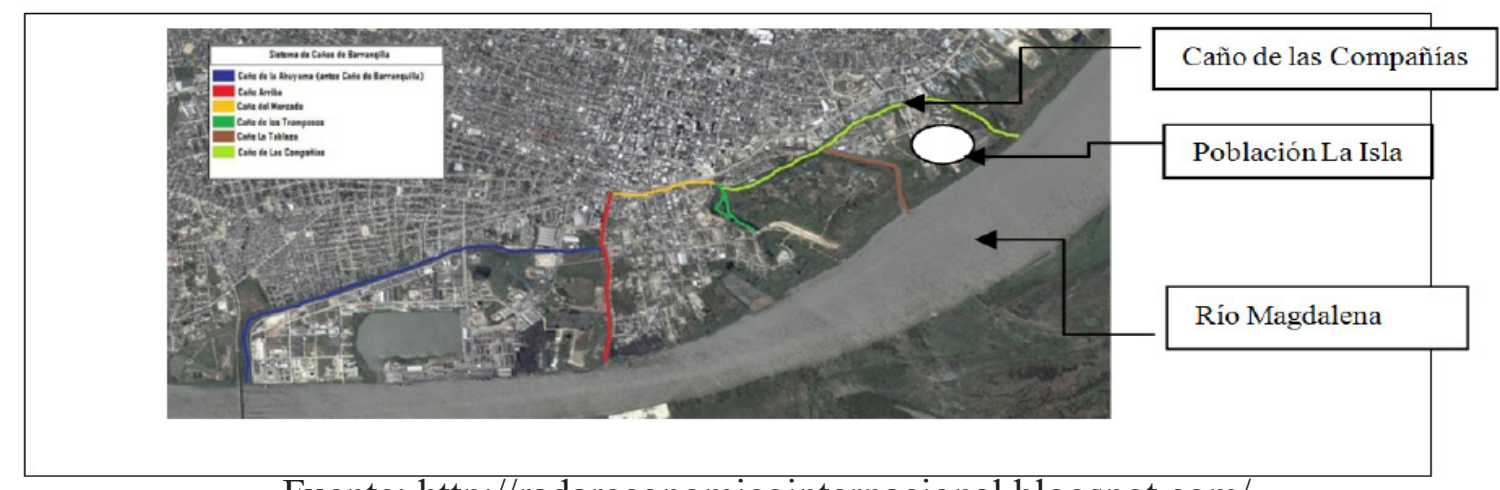

Fuente: http://radareconomicointernacional.blogspot.com/

De igual manera, los maestros en formación involucrados en el mismo fortalecen su sensibilidad humanística al descubrir que puede transformar vidas mediante la lúdica, la lectura, el arte y la enseñanza de la participación ciudadana, de la misma forma robustecen el pensamiento crítico frente a la vida y el respeto por la naturaleza que ayude a forjar conciencia ambiental y promover desde otros lenguajes y escenarios de aprendizaje, la importancia que tiene la interacción hombre-naturaleza, así como sus efectos en el medio ambiente y en el desarrollo socioeconómico" (Diaz, 2015). Lo que permite contribuir al fortalecimiento de la relación hombre - sociedad - naturaleza, para reinventar los espacios como lugares de vida y de paz.

La intervención a la comunidad, se articula a la labor de Proyección Social del programa Licenciatura en educación Básica Primaria de la Universidad de la Costa, pues se llega a la población de la Isla la cual es una zona de difícil acceso y un barrio de invasión.

\section{2.- FUNDAMENTO TEÓRICO}

\section{La lúdica como dinamizador para reinventar los espacios}

Para la intervención de los espacios y reinventarlos como lugares de vida y paz, se utiliza la lúdica como estrategia, la cual según Baquero permite actuar dentro de un escenario imaginario que el niño asuma un rol específico. Esto ratifica que las actividades lúdicas son una manera de actuación donde se vincula lo cognitivo con acciones hacia el entorno social articulando el aprendizaje, la participación en un contexto y la movilización de las funciones ejecutivas superiores de su estructura mental. 
De esta manera que en conjunto el juego y la lúdica generan espacios agradables de aprendizaje y de moldeamiento de conductas, transforman el ambiente hostil en un espacio acogedores e interesantes para la comunidad, es decir que lo que allí se realiza no está dependiendo del cumplimiento de reglas, sino que abre la oportunidad de comunicación y participación en su propio aprendizaje. Así mismo las relaciones sociales, la afirmación de roles, el liderazgo, la toma de decisiones, la autoestima, las habilidades de pensamiento y las habilidades motrices, son algunos aspectos que se desarrollan favorablemente a través del juego y la lúdica.

Por otro lado, la lúdica como experiencia cultural es una dimensión transversal que atraviesa toda la vida, no son prácticas, no son actividades, no es una ciencia, ni una disciplina, ni mucho menos una nueva moda, sino que es un proceso inherente al desarrollo humano en toda su dimensionalidad psíquica, social, cultural y biológica, desde esta perspectiva, la lúdica está ligada a la cotidianidad, en especial a la búsqueda del sentido de la vida y a la creatividad humana. Jiménez (2002) y se caracteriza por ser un medio que resulta en la satisfacción personal a través del compartir con otro.

Así mismo, Medina (1999), considera que las actividades dirigidas desde la lúdica, permiten crear unas condiciones de aprendizaje mediadas por experiencias gratificantes y placenteras, a través de propuestas metodológicas y didácticas innovadoras, diferentes a las convencionales en las que se desarrollan los pilares fundamentales de la educación: aprende a aprender, aprender a pensar, aprender a hacer, aprender a ser y aprender a convivir. Por tal motivo la lúdica promueve al desarrollo y la autoexpresión que le permiten al individuo fortalecer su personalidad y está a la vez contribuye directamente al entorno socio-educativo y potencializa la evolución cognitiva de la comunidad.

De la misma forma, los lineamientos Curriculares de Educación Física, recreación y deporte (2000), en los apartes donde aborda sobre el desarrollo del estudiante, relaciona la lúdica con la recreación y considera la primera como la posibilidad expresiva, formadora de sensibilidad, comunicación e integración cultural y social y como espacio de encuentro con la naturaleza, la cultura y las propias potencialidades individuales". En este sentido se relaciona con aquello que refuerza las formas de convivencia. Es decir, la lúdica es una manera de darle sentido, significación y transformación del juego en diferentes realidades posibles. Por ello la lúdica no se limita a espacios limitados de la escuela o del tiempo libre, sino que se proyecta a distintos espacios de la existencia y entorno social.

En este mismo sentido Romero (1992), afirma y da reconocimiento a la lúdica dentro del uso del tiempo libre como experiencia social y cultural y le atribuye al juego su máxima expresión, reconociéndolo como un mediador de las relaciones entre los hombres que abandona el valor instructivo y se impone la espontaneidad, de acuerdo con este, el juego no es una forma 
de reproducir los sentidos o regulaciones sociales, sino una manera de producir sentidos y flujos en los cuales la cultura se reactualiza, es decir, es leída como un presente que es exteriorizado.

En este orden de ideas, se puede considerar que la lúdica no es una estrategia establecida como tal, sino algo innato de cada persona, son acciones que se realizan a diario y que permiten expresarse con libertad. Por ello esta herramienta es de vital importancia en la intervención a la comunidad, porque son actividades al aire libre, en un contexto social, que permite la interiorización de cada uno de los mensajes que se quiere transmitir, representa más que una herramienta metodológica, un redimensionamiento del papel del individuo en el contexto formativo del niño, el joven y el adulto, que incide positivamente en su forma de ver, actuar y percibir el mundo. (Moreno, 2003)

Por todo lo anterior, se concluye que la lúdica motivada y estimulada de manera adecuada y procesual se convierte en una estrategia que puede ser implementada en procesos de rehabilitación, de atención integral de manera individual, grupal y de trabajo familiar, esta no discrimina y acepta al ser humano, porque reconoce la potencialidad y el talento, materializado por medio de la detección oportuna de intereses, necesidades y satisfactores lúdicos que faciliten procesos transformadores. (Moreno, 20003)

\section{Ilustración 2. Estudiantes de V semestre L.E.B.P Universidad de la Costa}

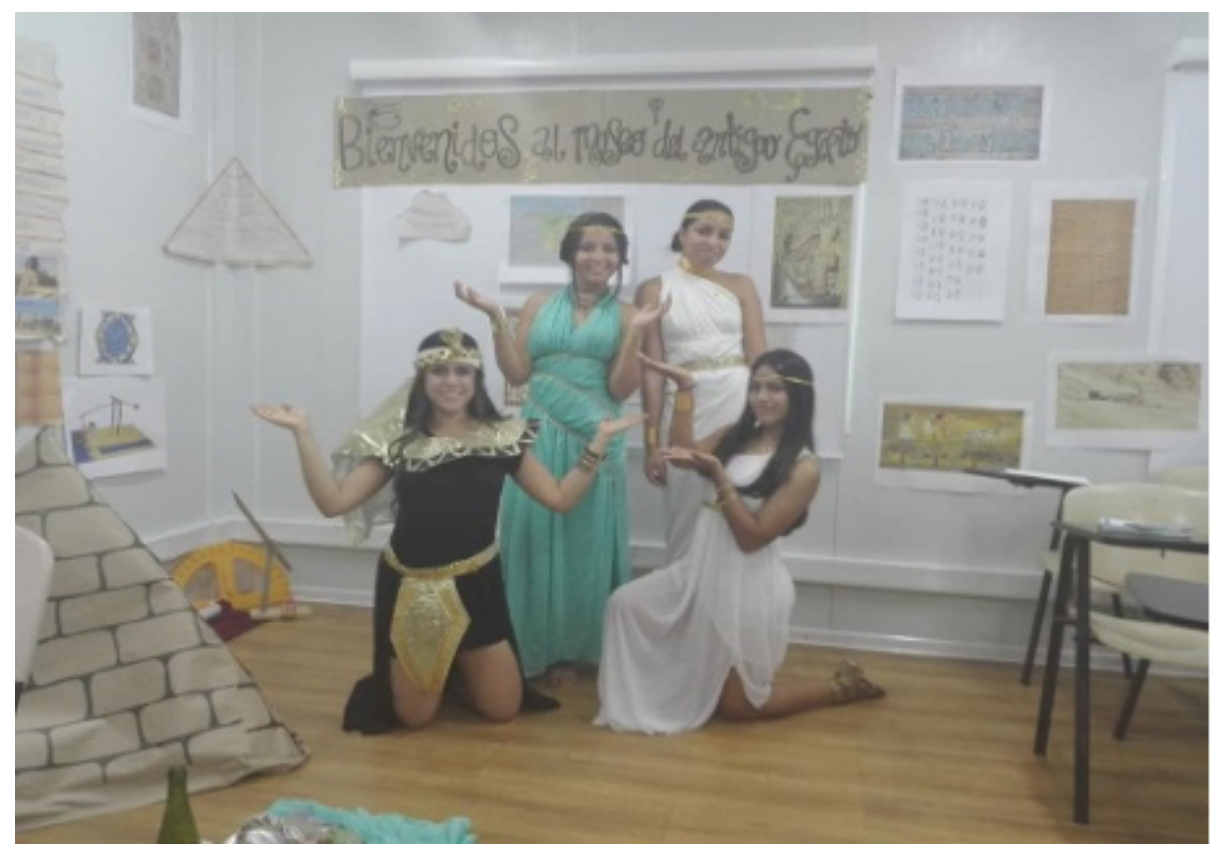

Fuente: Elaboración propia, (2016) 
Reinventando los espacios como lugares de vida y paz

Para darle sentido a reinventar los espacios, se significará desde la palabra reinventar, que según la definición del diccionario de Oxford, significa volver a inventar, es decir volver a crear en los espacios vulnerables una cultura de paz, esperanzadora que trasciende en la sociedad a partir de momentos de aprendizaje que mejoran la calidad de vida de la población de tal forma que se pueda como señalan Boqué \& otros ( 2014) articular de manera transversal la paz con justicia social, desarrollo, derechos humanos, democracia y gestión positiva de conflictos.

Reinventar los espacios busca desde su génesis, en los maestros en formación, la adopción de modos de vida que sean compatibles con la sostenibilidad adquirida, mediante la adecuada exploración, utilización, uso y manejo de los recursos naturales, aportando así una nueva visión de mundo y una oportunidad de vida, para que a partir de estos puedan sensibilizar a las comunidades vulnerables sobre la conciencia del cuidado del medio ambiente, la importancia de los escenarios de paz y la inteligencia emocional fortaleciendo de esta manera la relación hombre - naturaleza.

Para fortalecer la cultura ambiental en estos escenarios, se parte del análisis de la situación en el contexto mundial, donde se hace evidente la falta de conciencia sobre el mantenimiento de su entorno de manera responsable, su uso y su sostenibilidad a largo plazo. Se vive actualmente, en una "cultura de cemento", sin importar los daños al ecosistema, el medio ambiente, la capa de ozono y todos aquellos perjuicios colaterales que surgen desde el mal manejo de los recursos, el inadecuada tratamiento los desechos y la modificación inapropiada del medio ambiente.

A partir de estos, busca que los individuos y las comunidades puedan comprender la complejidad del daño que se le ha causado al ambiente que ha brindado la naturaleza misma desde su creación y el establecido por el ser humano como parte de su desarrollo cultural siendo resultado de la interacción de los factores biológicos, físico-químicos, sociales, económicos, políticos y culturales, para que se adquieran los conocimientos, valores, actitudes, destrezas y habilidades que les permitan participar de manera responsable, ética, afectiva en la prevención de la problemática del desarrollo sostenible de manera solidaria y responsable.

Con respecto a la problemática ambiental de orden mundial, Obama (2016), reconoció que todas las naciones, deben estar alertas al cambio climático al explicar que en Alaska los glaciares se están derritiendo a un ritmo "sin precedentes" y que el mar se está "tragando" a las aldeas.

Estas palabras del presidente de Estados Unidos, son una muestra de la preocupación que existe en los mandatarios del mundo ante la crisis ambiental mundial y un llamado para que se fortalezca la cultura ambiental, que trascienda como parte de la formación integral del ser.

Desde la epistemología antropocentrista, el hombre desde el principio de los tiempos, se ha relacionado con la naturaleza, la ha tomado para su disposición y por tanto ha abusado de los re- 
cursos naturales disponibles. Vélez (2016). Estas razones han llevado a un proceso de reflexión en el que se promueva una estrategia de convivencia con la naturaleza que permita concientizar sobre el uso racional y sustentable de los recursos humanos.

Algunos de los problemas ambientales, del mundo contemporáneo, según el mismo Vélez son: El agotamiento de la capa de ozono (debido al uso excesivo de clorofluorocarburos (CFC)) el agotamiento de los recursos (dada la sobreexplotación de los recursos naturales), la agricultura intensiva ( a causa del sobre pastoreo, el exceso de riego, el monocultivo), el aumento de especies en vías de extinción (disminución de los polinizadores, blanqueamiento de los corales, especies invasoras, caza ilegal, etc.), cambios globales(calentamiento global, oscurecimiento global, disminución de los combustibles fósiles, aumento del nivel del mar, etc.), consumismo (creencias y prácticas que promueven y estimulan el consumo excesivo de pesca, bosques, minerías), entre otros.

Además de lo anterior según Rivera (1999), se suma a la contaminación la perturbación de la estructura de las relaciones de los seres vivos y el medio en que se desarrollan, la deficiente gobernabilidad ( falta de políticas claras, poca aplicabilidad de las leyes), degradación del medio ambiente y del suelo, poco uso de energías renovables, los residuos, la superpoblación, valores sociales cambiantes que son algunas de las consecuencias del mal uso del suelo y los recursos por parte del hombre".

El desconocimiento del papel del hombre en el mundo con respecto a su relación con otros hombres, la naturaleza y la sociedad dificulta la interacción del hombre como individuo y con los miembros de los grupos sociales, generando estilos de vida insostenibles, ya que se generan procesos de competitividad destruyendo los valores, las creencias, las costumbres, las relaciones haciendo uso inadecuado de los recursos, generando relaciones hostiles y de poca participación y conformismo haciendo del desarrollo un proceso desigual para las sociedades humanas y perjudicial para los sistemas naturales. (Bayón, 2006).

En este orden de ideas, desde la intervención se hace necesario conocer las diferentes políticas ambientales para la preservación del medio ambiente, despertar el sentido de pertenencia de su entorno, la ciudad la región el país y el mundo, para que la comunidad cuente con las bases fundamentales claras que ayuden desde el contexto a la concientización de una cultura ambiental haciendo de estas un aprendizaje significativo extrapolado al contexto real, como alternativa para preservar el medio ambiente y garantizar la supervivencia de las especies.

En consecuencia, se pretende la construcción de una cultura ambiental a tono con la realidad nacional e internacional fortaleciendo la concientización de esta desde los procesos educativos para que se refleje en los procesos sociales y culturales, como pilar del desarrollo sostenible.

Una cultura ambiental que propenda en palabras de Marulanda \& Luna (2003) por la vida 
que todos merecemos, donde se hable no solo de las especies en vida de extinción o de como disminuir la contaminación, sino también del bienestar de la sociedad, de una cultura que crezca en el respeto, de calidad de vida para todos los integrantes de la realidad: los factores bióticos, abióticos, históricos, culturales. Es decir una cultura ambiental que ayude a reconstruir la relación hombre - sociedad - naturaleza, reinventando los espacios como lugar de vida y de paz en armonía sistemática de todos los elementos que en ella confluyen.

De la misma forma, teniendo en cuenta la intencionalidad de la intervención, se propende por concientizar y fortalecer participación ciudadana, que genere una cultura de paz, que supone ante todo un esfuerzo generalizado para modificar mentalidades y actitudes con ánimo de promover la paz. Significa prevenir y transformar los conflictos que puedan engendrar violencia y restaurar la paz y la confianza en poblaciones que emergen de la guerra. Pero su propósito trasciende de los límites de los conflictos armados para hacerse extensivo también a las escuelas y los lugares de trabajo del mundo entero, los parlamentos y las salas de prensa, las familias y los lugares de recreo.

Forjar una cultura de paz es hacer que los niños y los adultos comprendan y respeten la libertad, la justicia, la democracia, los derechos humanos, la tolerancia, la igualdad y la solidaridad. Ello implica un rechazo colectivo de la violencia. E implica también disponer de los medios y la voluntad de participar en el desarrollo de la sociedad. (Documento de la UNESCO hacia una Cultura de Paz, 1998).

\section{Competencias emocionales}

Las competencias emocionales según el autor Jiménez (2011), son consideradas un saber hacer contextualizado, que todo ser humano adquiere por vía educativa (formal- no formalinformal), en un determinado contexto, son diferentes en cada contexto y que solo es posible identificar y evaluar en la acción misma. Ahora bien, al referirse al término de competencia, se hace referencia a cada una de las capacidades o habilidades, destrezas o conocimientos que posee el ser humano para desenvolverse de forma efectiva en su vida.

Por su parte el psicólogo Bosque las habilidades o competencias emocionales el Psicólogo argumenta que las competencias emocionales, hacen parte de las capacidades adquiridas, por medio de las experiencias y la formación, estas son aprendidas y se pueden fortalecer desarrollándolas.

Por su parte los autores Bisquerra y Pérez (2013), argumentaron que las competencias emocionales son entendidas como un subconjunto de las competencias personales y se pueden clasificar en conciencia emocional, regulación emocional, autonomía emocional, competencia social, habilidades de vida y bienestar. Resaltando así la aplicación de las competencias 
emocionales en la empresa, la salud y la educación. Aportando significativamente al objeto de estudio las competencias de este siglo que se pueden expresar en los siguientes términos:

Desde la conciencia emocional, se adquiere conocimiento de las propias emociones y de las emociones de las personas que están en el entorno.

Las emociones se pueden regular para manejarlas de forma apropiada. Tomando así conciencia de la relación entre emoción, cognición y comportamiento.

La autonomía emocional fortalece la autogestión personal, autoestima, actitud positiva ante la vida, responsabilidad y capacidad para analizar críticamente las normas sociales.

Competencia social que facilita el manejo de las buenas relaciones con otras personas.

Competencias para la vida y el bienestar: es la habilidad para adoptar comportamientos apropiados y responsables para afrontar satisfactoriamente los desafíos diarios de la vida, ya sean estos privados, profesionales o sociales".

Estas competencias permiten organizar la vida de forma sana y equilibrada, facilitando experiencias de satisfacción o bienestar. Es por esto que se considera que, para poder llegar a tener una inteligencia emocional, se deben poseer competencias que sirvan para toda la vida.

Por otra parte, Gardner (2004) en su Teoría de las inteligencias múltiples introdujo la idea de la inteligencia interpersonal y la inteligencia intrapersonal, pues son las asociadas a las competencias e inteligencia emocional. En cierta forma, la inteligencia emocional está formada por estas dos inteligencias, debido a que la inteligencia interpersonal es una de las facetas de la personalidad que incluye la capacidad de liderazgo, resolver conflictos y análisis social. El análisis social consiste en observar a los demás y saber cómo relacionarnos con ellos de forma productiva. Otra forma de expresar la inteligencia interpersonal es poniendo el énfasis en la habilidad de comprender a los demás: lo que les motiva, cómo trabajan, cómo trabajar con ellos de forma cooperativa también puede ser considerada como inteligencia social. (Zirkel, 2000, Topping, Bremmer y Holmes, 2000; Topping, Holmes y Bremmer, 2000; Cherniss, 2000; etc. citado por Bisquerra 2009).

En cuanto, a la inteligencia intrapersonal se refiere a la capacidad de formarse un modelo preciso de sí mismo y de utilizarlo de forma apropiada para interactuar de forma efectiva a lo largo de la vida, la cual tiende a coincidir con la inteligencia personal (Sternberg, 2000; Hedlund y Sternberg, 2000 citado por Bisquerra 2009 ) aspectos que deberían esencial en la escuela, debido a que este tipo de inteligencias interviene en las decisiones esenciales de la vida: elegir una profesión, con quién casarse, dónde vivir, etc. (Bisquerra 2009).

Más tarde, Goleman (2013) menciona que la llamada motivación hace parte de la competencia emocional, desde esta perspectiva, el autor considera que la motivación proviene de cuatro fuentes principales: el individuo mismo, los amigos, la familia y colegas, complementados con 
un mentor emocional, ya sea real o ficticio; y el propio entorno, es decir, aire, luz, sonido, u otros objetos de motivación.

Los aportes de los teóricos citados anteriormente permiten tener claridad de la importancia que tiene formar en competencias emocionales en la relación hombre-sociedad- naturaleza, teniendo claro que la forma en cómo se resuelven los conflictos, se convierte en oportunidad de formación tanto para la competencia personal como para la vida en sociedad.

Los conflictos que se presentan en la cotidianidad deben ser resueltos bajo el prisma de oportunidades y aprendizajes, poniendo en juego el manejo de las emociones. Por consiguiente, cabe mencionar la influencia de la inteligencia emocional que los científicos Salovey y Mayer (2001), definen como parte de la inteligencia social y que aborda la capacidad de controlar los sentimientos y las emociones propias, así como las de los demás. Si bien, resulta imposible medir cuantitativamente las características sociales y de personalidad, es por esto que se considera sumamente importante estimular con la misma exigencia las capacidades emocionales e intelectuales.

\section{3.- MÉTODO}

\section{Diseño}

El presente estudio, se hace desde el enfoque de la investigación acción participativa, que enfatiza en el aprendizaje colectivo de la realidad basado en un análisis crítico con la participación activa de los actores implicados, los cuales analizan la realidad y orientan las intervenciones para estimular la práctica transformadora y el cambio social.

El abordaje se hace desde un paradigma cualitativo, que de acuerdo con Bonilla y Rodríguez (2005), este tipo de investigación se orienta a profundizar casos específicos y no a generalizar. Su preocupación no es prioritariamente medir, sino cualificar y describir el fenómeno social a partir de rasgos determinantes, según sean percibidos por los elementos mismos que están dentro de la situación estudiada. (p.60).

El alcance es descriptivo el cual según Tamayo este tipo de estudios, permite analizar, describir, registrar e interpretar la realidad de la naturaleza actual, y la composición o proceso de los fenómenos. El enfoque se hace sobre conclusiones dominantes o sobre grupo de personas, grupo o cosas, se conduce o funciona en presente.

Alcanza un diseño no experimental el cual permite realizar el estudio sin la manipulación deliberada de variables, sólo se observan los fenómenos en su ambiente natural y después se analizan. (Hernández, Batista y Fernández, 2014, p. 149) y de campo que consiste en la recolección de datos directamente de los sujetos investigados, o de la realidad donde ocurren los hechos (datos primarios), sin manipular o controlar variable alguna, es decir, el investigador 
obtiene la información, pero no altera las condiciones existentes.

Las técnicas e instrumentos implementados para acceder a la información necesaria y obtener la información durante el estudio fueron: la observación la cual permitió acceso directo al lugar de estudio ( Isla La Loma), a partir del contacto inicial se hizo la captación para percibir las características o manifestaciones presentes que van a permitir determinar los temas de abordaje, de la misma forma se utilizó la entrevista que permitió la interacción personal con otras personas para obtener información y analizar con mayor profundidad la realidad y se realizaron sesiones en profundidad en las que a partir de la implementación de la lúdica se propicia el flujo de información para complementar la investigación social, los datos obtenidos se sistematizaron en diarios de campos y en registros fotográficos.

La población participante hace referencia a los actores del sector vulnerable de la Islita Caño que tienen 500 habitantes aproximadamente representados entre: amas de casa, madres gestantes, vendedores ambulantes, desempleados, lactantes, niños, niñas, jóvenes, adultos y adultos mayores que conforman la comunidad. El muestreo de la población es aleatorio para que todos los miembros de la comunidad pudieran participar de la intervención con las siguientes características: Niños, niñas y jóvenes adolescentes entre los 0 y 15 años y madres gestantes o lactantes.

\section{Procedimiento}

El presente estudio se realiza en fases de abordaje que se describen de la siguiente forma:

Fase 1 o fase Inicial: Selección de la población vulnerable para el proceso de intervención; el cual se lleva a cabo a través de un contacto inicial con la comunidad del sector Isla de la Loma se hizo una observación directa del sector y una caracterización socio- demográfica, que permitió reconocer las características de dicha población y seleccionar los temas de abordaje para la intervención. Una vez terminada esta etapa inicial el grupo investigador procedió a realizar un proceso de reflexión con base a lo recopilado.

Fase 2: Representada en la construcción de un plan de acción, desde la sesión en profundidad para hacer intervención con las actividades lúdicas como dinamizador del proceso para reinventar los espacios como lugares de vida y de paz con la población muestra participante del proyecto, los cuales son 20 madres entre gestantes y lactantes y 65 niños entre los 0 y 12 años y 15 adolescentes entre los 13 y 15 años de edad.

Se programa la ejecución de talleres experienciales, con una duración de 3 horas los fines de semana. Para su diseño se partió de la conciencia sobre la importancia de la cultura ambiental, la paz y la inteligencia emocional en las dimensiones del ser humano y en su valioso aporte dentro de los procesos de convivencia y desarrollo de la comunidad. 
Fase 3: consiste en la Ejecución del Plan de Acción, aquí se retoma la lúdica como una estrategia novedosa y eficaz para desarrollar la capacidad de pensar, como medio de expresión; tanto en el plano físico, como en el cognitivo, social y psicológico. Así mismo, a través de la realización de los talleres, se propició el proceso de apropiación y fortalecimiento de la cultura ambiental, la paz y la inteligencia emocional en la población vulnerable abordada.

Por lo tanto, el diseño de los talleres lúdicos para la reinvención de los espacios, incentivó en los niños, niñas, jóvenes y madres la curiosidad y la motivación por participar con libertad; propiciando a su vez la interacción con el entorno cotidiano, de la misma forma complementó está fase con la técnica de observación participante; por medio de la cual los investigadores se involucraron en los procesos de aplicación de la lúdica con las población seleccionada, posibilitando espacios de interacción social que les permitió reconocer en las voces de los niños, niñas, jóvenes y madres en su desenvolvimiento diario.

La ejecución y el trabajo de campo se componen de tres fases que consistieron en la intervención para fortalecer la cultura ambiental, la participación ciudadana y la inteligencia emocional. Una vez recopilada y analizada la información se construye el documento objeto de este trabajo.

Fase 4: corresponde a la fase de cierre de la Investigación, en la cual se sistematizan, y verifica la incidencia de la lúdica y las temáticas seleccionadas, para poder determinar desde la reflexión si la dinamización desde la lúdica, coadyuvó a la potenciación de las transformaciones esperada en la población vulnerable.

Para el proceso de finalización de la intervención se propició un espacio de reflexión donde participaron todos los actores y el grupo investigador. La información obtenida se recopiló en el diario de campo y en registros digitales como: videos y fotografías.

Una vez terminada la intervención, se analizó y reflexionó acorde a los resultados obtenidos por el grupo de actores durante el primer y tercer momento, estableciéndose así las diferencias relacionadas con el antes y después de la implementación de los talleres; de esta forma se determinó si la lúdica permite reinventar los espacios como lugares de vida y paz de la población vulnerable.

\section{4.-RESULTADOS}

Los alcances del proyecto se abordan en dos etapas:

Fase 1: El contacto con la población vulnerable del sector Isla de la Loma se hizo a través del convenio con la Biblioteca Infantil Piloto, a través de la entrevista que se le hizo a los funcionarios y que permitió identificar un contexto de aproximadamente 500 familias en condición de vulnerabilidad, carente de servicios públicos y con condiciones económicas extremadamente bajas o nulas, un sector con poca intervención del estado, cerca al caño de los Tramposos, sien- 
do un sector de aguas altamente contaminadas.

Para llegar a La Islita existen dos caminos: uno por tierra y otro por agua, atravesando el caño de las Compañías, sobre la ubicación de este sector la historia de Barranquilla, señala según Angulo (1992):

A fines del siglo XIX en la zona de la antigua Aduana, enfrente a La Islita, un ingeniero de nombre Antonio Luis Armenta, responsable de la construcción de una línea del tranvía hizo un importante descubrimiento de urnas cinerarias que daban testimonio de un viejo cementerio indígena. Circunstancia que explica la presencia temprana de seres humanos mucho antes de la llegada de españoles a América. El informe Armenta, fue divulgado en un libro publicado en 1942, titulado Colombia de norte a sur y fue escrito por el arqueólogo español, José Pérez de Barradas. Esta obra inspiró a Carlos Angulo Valdés, científico costeño del siglo XX, a adelantar estudios sobre los primeros asentamientos de seres humanos en diferentes lugares del perímetro de lo que hoy es Barranquilla. Seguramente el caño de las Compañías era aprovechado para la navegación de vapores que atracaban a la Intendencia Fluvial (adyacente a La Islita), reflejando con ello un activo comercio de la ciudad.

Para llegar a la Islita el grupo por tierra, ruta que hoy es posible por la construcción de la Avenida del Río, una larga carretera que se extiende paralela al rio Magdalena y que contribuye a mejorar la movilidad y el tránsito en este sector de Barranquilla

La Islita es un sector de origen sedimentario. Los ríos de gran caudal, como es el caso del Magdalena, arrastran grandes cantidades de sedimentos dando origen a la formación de islotes a lo largo de su curso. De igual manera influyó en su génesis toda la sedimentación que también arrastran los caños en particular el de Las Compañías. Hoy en día vive una humilde comunidad, sus viviendas han sido construidas en madera y los techos de láminas de zinc. En el barrio llamado la Islita no existe una institución educativa, los niños deben cruzar todos los días el caño de Las Compañías para ir a recibir sus clases en la escuela del barrio Barlovento, barrio que queda al frente de la Islita, con el riesgo de que por alguna imprudencia pueda hundirse la canoa y ocurra una calamidad.

Por otro lado, muchos padres de familia prefieren no asumir el riesgo de atravesar a sus hijos en la canoa, por eso decidieron que era mejor no ponerlos a estudiar; una cantidad considerable de niños están desescolarizados porque viven en una pobreza extrema que no solo es económica. Aquí tampoco existe un puesto de salud, circunstancia que unida a bajos niveles educativos explica el por qué hay un alto índice de mujeres embarazadas jóvenes que, a pesar de su avanzado grado de gestación, aún no han asistido a su primer control prenatal. Es muy notable que este lugar no fuera construido de manera planificada, pero de igual manera son familias que piden y merecen como colombianos la presencia del Estado. 
En cuanto a la seguridad, los habitantes de esta comunidad se han ideado un sistema de alarmas en conjunto con la Policía Nacional. De tal manera que ante cualquier situación de amenaza o sospecha que ponga en riesgo la integridad de la comunidad activa las alarmas y toda la comunidad se hace presente para ahuyentar la persona que pretende robarles su tranquilidad y es expulsado inmediatamente.

En otro orden de ideas, el abastecimiento de agua, la toman a través de red de tubos improvisados que la misma comunidad ha construido, y se encuentra conectada a un tubo madre cercano que la lleva al barrio. De al igual forma ocurre con el alcantarillado y el servicio de electricidad; hasta hace poco fue colocado un medidor comunitario y aspiran que en poco tiempo les normalicen el servicio de energía porque éste es bastante inestable.

En cuanto a las calles ninguna esta pavimentada y en época de lluvias es difícil trasladarse de un lado a otro porque se convierten en lodazales. Existe una sola tienda que satisface las demandas de consumo de sus moradores. En alguna ocasión mientras el proyecto se ejecutaba hubo necesidad de solicitarle que bajaran el volumen del equipo de sonido porque desde muy temprano colocan música, circunstancia que dificulta por momentos que los niños gocen de un ambiente en silencio que les permita concentrarse y consagrarse al mundo de la lectura o participen activamente en las respuestas, a aquellas preguntas de sus maestros.

Sobre las actividades económicas, esta comunidad se dedica a la agricultura y a la pesca. Algunos de sus habitantes han construido estanques internos donde cultivan mojarra roja y cachamas. Tienen sembrados de plátanos y cocoteros que una vez han recogido la cosecha la venden en el mercado de Barranquilla. Otras personas se trasladan a trabajar en las casas de familia en la ciudad o en actividades informales en el mercado, siempre con la dificultad del traslado antes de que anochezca.

Fase 2: En esta etapa, desde el aula de clases se busca sensibilizar a los maestros en formación de $\mathrm{V}$ semestre, en los escenarios propuestos tales como: población vulnerable, la educación y la cultura de paz, el desarrollo sostenible como artífice del respeto y cuidado del medio ambiente y la inteligencia emocional.

Para esto, hace todo el abordaje de la problemática mundial del medio ambiente y lo lleva a reflexionar sobre la construcción de las relaciones de las civilizaciones con el medio, analizando que en un principio el hombre encontró todo lo necesario brindado por la naturaleza, pero estas relaciones se deterioraron cuando en la edad moderna el hombre al descubrir la razón se cree dueño y señor, empezando a intentar dominar la naturaleza, olvidando que solo existe este lugar con las condiciones para ser habitado, así mismo se hace todo el proceso de concientización y fortalecimiento de las competencias en democracia, competencias ciudadanas y participación ciudadana 
De este proceso de sensibilización en el aula, se estructuró una recopilación de historias, compiladas en CUColibro, que es una creación de los maestros en formación der V semestre de la Universidad de la Costa en el que se encuentran historias de género literario (fábulas, leyendas, mitos) o género lírico (poesías, canciones), así como rondas infantiles. Cada construcción textual, tiene como intencionalidad, dejar una enseñanza o moraleja a los niños y niñas acerca del cuidado del medio ambiente y la formación en participación, los cuales fueron narrados en el proceso de sesiones a profundidad, una vez terminada esta etapa inicial el grupo investigador procedió a realizar un proceso de reflexión con base a lo recopilado.

Ilustración 3. Mascota y lema del proyecto, creación estudiantes V semestre de Licenciatura de Educación básica primaria. Universidad de la Costa- CUC-

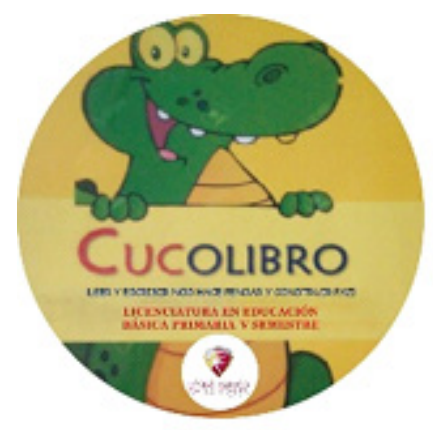

Fuente: Elaboración propia, (2016).

Fase 3: En esta etapa los maestros en formación contextualizan y extrapolan las vivencias del aula a los escenarios posibles donde se pueda reinventar los espacios como lugares de vida y de paz (zonas vulnerables, parques, teatros, instituciones educativas con problemáticas, etc.).

En estos momentos, el abordaje se está haciendo directamente en la población de la Isla, para esto los estudiantes preparan con mucha dedicación, en coordinación con la Biblioteca Infantil del Caribe el plan de acción de cada sesión, que consiste en una actividad inicial de bienvenida a la comunidad, luego se organizan por grupos de edades e intereses para trabajar las temáticas propuestas. Casi siempre el encuentro con esta comunidad ocurre cada sábado, momento en el cual, se organizan actividades educativas, lúdicas y recreativas con los niños de ese sector, se inicia con la concientización sobre entornos limpios y agradables para el aprendizaje, además de conocer los colores y las canecas del reciclaje a través de una fábula.

De la misma forma, a través de las actividades lúdicas, se busca cambiar el concepto que estos niños y niñas tienen acerca de la interacción con el medio ambiente. Entender que la naturaleza es un ser preciado que se ha dado a los seres humanos para hacer buen uso de ella 
y sobre todo cuidarla y amarla, creando un nuevo concepto de ética donde no está presente el pensamiento de dominación y mucho menos el de explotación para el beneficio de unos pocos. Debemos sentir la naturaleza como el hogar común.

Siendo un sector vulnerable y espacio al aire libre, se necesita de la colaboración de la comunidad para la atención y ubicación de los miembros de la comunidad que participan del proyecto, para esto la señora Marta (vecina de la comunidad), presta las sillas en las que se ubican los niños y niñas. Algunos de ellos hay que buscarlos a sus casas porque sufren de alguna discapacidad para movilizarse, y cada maestro sabe cuáles son sus estudiantes. De tal manera, que para garantizar su asistencia es necesario ir por ellos para que participen activamente y posteriormente llevarlos a sus casas una vez concluida la actividad.

Los maestros en formación, ejecutan las actividades lúdicas que llevan preparada desde una fábula o cuento del texto CUColibro, cada lectura tiene la intención de dejar una enseñanza acerca del cuidado del medio ambiente, conocer acerca de sus derechos como ciudadanos o aprender cómo resolver los conflictos que se les pueden presentar, en forma pacífica, la animación y recreación de la lectura la hacen los maestros haciendo pausas para preguntar acerca del texto leído y la enseñanza.

De la misma forma, se juego de roles sobre los derechos humanos, ya que la educación en derechos humanos otorga especial significado a la misma naturaleza humana. En gran parte de esta actividad se nota en los niños (as), desconocen sus derechos o simplemente no los vivencian y por tal motivo es necesario hacer que ellos los vivan y los plasmen para convertir los derechos en parte de la vivencia de esta comunidad.

Mientras los niños(as) están en su espacio de lectura y recreación, a las madres se les enseña algunas manualidades que les sirva para una actividad productiva o, por ejemplo, cuidados necesarios para la atención del bebé en el caso de las madres gestantes primerizas. A otras se les enseña acerca del uso responsable y adecuado de los métodos anticonceptivos debido a que -algunas de ellas son jóvenes y con bajo nivel de escolaridad- tienen hasta cinco o seis hijos pequeños. De igual forma a toda la comunidad y viendo la dificultad para la obtención del agua y el fluido eléctrico se les hacen recomendaciones acerca del buen uso de estos.

Como impacto social, se observa el alcance del objetivo del proyecto que es reinventar espacios, que transcurren en la monotonía del diario vivir sin dedicar un espacio a la reflexión, a la lectura, pero sobre todo a fortalecer esas relaciones interpersonales que enriquecen y permiten desarrollar y fortalecer ese humanismo que está deteriorado por el incesante transcurrir apresurado del tiempo, es decir como lugares de paz.

Cabe resaltar que la satisfacción más grande del maestro en formación es saber que está contribuyendo a un espacio lúdico- recreativo en esta comunidad. 
Finalmente, para las actividades de despedida se organiza una fiesta donde se realiza la exposición de los dibujos de los niños(as), todas las manualidades de las madres y los maestros en formación de la Universidad de la Costa, CUC, dramatizaron la popular obra infantil "El renacuajo paseador" de Rafael Pombo, Lo más importante es que las conciencias iban llenas de aquella esperanza de volver a ver en el próximo semestre a esos niños quienes muestran una alegría sin causa, que logró contagiar con su ternura y espontaneidad. Pero ante todo lograron despertar en el maestro en formación el tacto o la sensibilidad pedagógica, que se define como el resultado de las relaciones interpersonales especialmente cuando el maestro trabaja con niños.

De la vivencia de las experiencias, se puede concluir que: La función de los maestros en formación consiste entonces, en crear a través de su tacto pedagógico un entorno de aprendizaje seguro y estimulante y ayudar a la comunidad a alcanzar un equilibrio entre las actividades. El maestro, es entonces un facilitador y no un proveedor de información, es quien debe tener el deseo de motivar en la comunidad la formación integral para la vida, desde el ser, el hacer, el convivir y el estar. Trascendiendo el acto pedagógico más allá de las aulas de clases, comprendiendo que la acción de educar es en todo momento y en el lugar que se necesite.

Es así como amplió el pensamiento de la inclusión y la diversidad siendo capaz de vivir la experiencia, jugar, enseñar y reinventar espacios de vida y de paz a los niños(as) y madres que encontraron en la Islita.

Los beneficios que ha generado de manera recíproca para las estudiantes de $\mathrm{V}$ semestre de licenciatura en Educación Básica Primaria y para la población beneficiaria en la Islita, son: Sensibilización sobre el impacto ambiental.

Fortalecimiento y compromiso de desarrollo sostenible

Transformación de historias de vida

Articulación de la lectura y la narración oral para fortalecer la relación hombre - sociedadnaturaleza

Recreación lúdico- pedagógica de la relación hombre- sociedad- naturaleza

La creación de CUColibro como logo del acompañamiento.

Leer y escribir nos hace pensar y con CUColibro lo vamos a lograr como lema.

\section{5.- CONCLUSIONES}

La implementación de la lúdica como dinamizador social para reinventar los espacios como lugares de vida y paz, permitió alcanzar las siguientes conclusiones:

Los niños, niñas, jóvenes y madres intervenidos lograron identificar la importancia de la cultura ambiental, los espacios de paz y la inteligencia emocional. 
El trabajo de campo y la observación directa permitió conocer con claridad las características de la población lo cual facilitó partir hacia la búsqueda de estrategias de fortalecimiento de la cultura ambiental, los espacios de paz y la inteligencia emocional.

La implementación de la lúdica se facilitó gracias a la variedad de recursos en desuso que ofrece el entorno cotidiano del sector Isla de la Loma.

Los talleres de intervención permitieron a los niños, niñas, jóvenes y madres de población vulnerable expresarse libremente y desplegar al máximo su criticidad y creatividad.

La lúdica es una técnica que dinamiza desarrollo de los procesos de intervención en los sectores vulnerables y garantiza el reconocimiento de la diversidad.

El individuo en la medida que se encuentra expuesto a actividades y procesos lúdicos, desarrolla su personalidad creadora, puesto que se destaca y logra un espacio reconocido por su manera de pensar y actuar, en la sociedad.

La lúdica se convierte en una técnica llamativa, curiosa e interesante para los individuos, ya que por medio de ello se logra brindar un ambiente de libertad y respeto.

A través de los talleres lúdicos se garantizó la calidad, equidad e igualdad en la formación de los individuos.

\section{REFERENCIAS}

Aguaded, M. Pantoja, M. (2015). Innovar desde un proyecto educativo de inteligencia emocional en primaria e infantil. Tendencias pedagógicas. Recuperado de https://revistas. uam.es/tendenciaspedagogicas/article/view/2122 Marzo 12 de 2020

Angulo, C. (1992).Contribuciones a la historia antigua de Barranquilla. Revista Huellas, 35 (fascículo), 7-8.

Bisquerra, R. (2013). Las competencias emocionales, Grupo de recerca en orientación Psicopedagogica.http://www.ub.edu/grop/wp-content/uploads/2014/03/ Orientaci\%C3\%B3n-psicopedag\%C3\%B3gica-y-educaci\%C3\%B3n-emocional.pdf

Boqué Torrermorell, Maria Carme, Pañellas Valls, Mercè, Alguacil de Nicolás, Montserrat, \& García Raga, Laura. (2014). La cultura de paz en la educación para la ciudadanía y los derechos humanos en los libros de texto de educación primaria. Perfiles educativos, 36(146), 80-97. Recuperado en 01 de mayo de 2020, de http://www.scielo.org.mx/scielo. php?script=sci_arttext\&pid=S0185-26982014000400006\&lng=es\&tlng=es. 
Cazallo, A.; Meñaca, I.; Lechuga, J.; Medina, H.; Uribe, C. y Barragán, C. (2019). Modeling foreign investment received in the Colombian oil sector during the period 1996-2016. https:/www.revistaespacios.com/a19v40n20/19402013.html

Colmenares, A. Investigación-acción participativa: una metodología integradora del conocimiento y la acción Recuperado de Dialnet-InvestigacionaccionParticipativa-4054232. pdf

Díaz Cutiño, R. (2015). Desarrollo Sustentable una oportunidad para la vida, tercera edición. México Df. Mac Graw Hill.

Echeverri, J. Hy Gómez, J. G. (2009). Lo lúdico como componente de lo pedagógico, la cultura, el juego y la dimensión humana. Recuperado de http://blog.utp.edu.co/areaderecreacionpcdyr/ files/2012/07/LO-LUDICO-COMO-COMPONENTE-DE-LO-PEDAGOGICO.pdf

Freire P. (1994). Cartas A Quien Pretende Enseñar. Editorial: Siglo XXI

Hernández, R., Fernández, C., y Baptista, P. (2014). Metodología de la investigación. México: Mc Graw Hill.

Gardner, H. (2004). Audiences for the theory of multiple intelligences. Teachers College Record, 106, 212-220.

Goleman (2013). Inteligencia emocional, California. Editorial Kairos

Jiménez, B. (2002). Lúdica y recreación. Colombia: Magisterio. Recuperado de http://wsp. presidencia.gov.co/Normativa/Leyes/Documents/LEY\%201732\%20DEL\%2001\%20 DE\%20SEPTIEMBRE\%20DE\%202014.pdf

Jiménez, C. (2011). Neuropedagogía, lúdica y competencias. Bogotá D.C. Colombia: Magisterio.

Medina G., C. (20 de Octubre de 1999). Gramática de la ternura Google Docs. Recuperado de https://docs.google.com/document/d/18Nq4S3fUUQVHST8Rsg264pD8JeYgDG4fjpw42 4rd2Hc/edit?pli=1

Ministerio de Educación Nacional Colombia (2013). Ley 1620 de 2013, Convivencia escolar. Bogotá (Colombia): Autor.

Ministerio de Educación Nacional (Sf). Lineamientos Curriculares de Educación física, recreación y deporte. Recuperado de http://www.mineducacion.gov.co/1759/ articles-339975_recurso_10.pdf 
Nuñez-Ríos, G., García-Mendoza, K., Castillo-Martelo, J. \& Niño-Jiménez, N.

Ministerio de Educación Nacional (2015). Decreto 1038 de 2015, reglamentación de cátedra de la Paz. Bogotá (Colombia): Autor. Recuperado de http://www.scielo.org.co/pdf/pml/ v8n2/v8n2a10.pdf

Miranda, L. (2013). Cultura ambiental: un estudio desde las dimensiones de valor, creencias, actitudes y comportamientos ambientales. Recuperado de http://www.scielo.org.co/pdf/ $\mathrm{pml} / \mathrm{v} 8 \mathrm{n} 2 / \mathrm{v} 8 \mathrm{n} 2 \mathrm{a} 10 . \mathrm{pdf}$

Núñez Ríos, G., Márquez Núñez, E., Guerrero Cuentas, H., Magdaniel Durán, M., \& Silvera Goenaga, B. (2019). La Metodología Lúdica Como Dinamizador De Las Conductas Prosociales. Lúdica Pedagógica, 1(30), 1-15. Recuperado a partir de https://revistas. pedagogica.edu.co/index.php/LP/article/view/11101

Rengifo, B. y otros (2012). La educación ambiental una estrategia pedagógica que contribuye a la solución de la problemática ambiental en Colombia. Recuperado de http://www.ub.edu/ geocrit/coloquio2012/actas/06-B-Rengifo.pdf

UNESCO (1999). Proyecto transdisciplinario de la UNESCO: Hacia una cultura de la paz. Recuperado de: http://unesdoc.unesco.org/images/0011/001177/117753So.pdf

/Dialnet-CulturaAmbiental-5012134.pdf.

Vergara, J. R. y Baena, F. (1992). Barranquilla, su pasado y su presente. Barranquilla (Colombia): Banco Dugand.

Zúñiga, M. (Año). Educación inclusiva relevancia de la sensibilidad pedagógica ante la diversidad educativa. Recuperado de:

http://www.adeepra.org.ar/congresos/Congreso\%20IBEROAMERICANO/ EDUCINCLUSIVA/RLE2370_Zuniga.pdf 